\title{
New algorithm for multiple pattern matching using least count of pattern
}

\author{
NITIPRASAD NAMDEORAO JAMBHULKAR* AND LOTAN KUMAR BOSE ${ }^{1}$ \\ Division of Social Sciences, Central Rice Research Institute, CUTTACK (ODISHA) INDIA \\ (Email : nitiprasad1@gmail.com)
}

\begin{abstract}
Rapid advances in genome research in the past have resulted in generation of large set of data for DNA and protein sequences from different prokaryotic and eukaryotic genomes. DNA is made up of four nitrogenous bases known as adenine $(A)$, guanine $(G)$, thymine $(T)$ and cytosine $(C)$. Pattern matching technique generally divides into two categories i.e. single pattern matching and multiple pattern matching. When it is required to find all occurrences of the pattern in the given string, it is known as single pattern matching. When more than one pattern are matched against the given string simultaneously, it is known as multiple pattern matching. The study of pattern matching is one of the applications in the field of bioinformatics. Many algorithms are available in literature for pattern matching. The purpose of pattern matching algorithm is to reduce the number of character comparisons. Hence, in this paper an algorithm Multiple Pattern Matching using Least Count of Pattern (MPMLCP) has been proposed for multiple pattern matching. The proposed algorithm has been compared with the available algorithm and it has been shown that the number of comparisons reduces over the available algorithms. SAS 9.3 software package has been used for calculating the number of comparisons.
\end{abstract}

Key Words : Pattern matching, Least count, Pre-processing, Algorithm, SAS software

View Point Article : Jambhulkar, Nitiprasad Namdeorao and Bose, Lotan Kumar (2015). New algorithm for multiple pattern matching using least count of pattern. Internat. J. agric. Sci., 11 (2) : 330-336.

Article History : Received : 01.04.2015; Accepted : 16.05 .2015

\footnotetext{
* Author for correspondence

${ }^{1}$ Crop Improvement Division, Central Rice Research Institute, CUTTACK (ODISHA) INDIA
} 